\title{
ANÁLISE DO COMPORTAMENTO DE OBSERVAÇÃO
}

\author{
ANALYSIS OF OBSERVING BEHAVIOR
}

\section{Candido Vinicius Bocaiuva Barnsley Pessôa e Tereza Maria de Azevedo Pires Sério PONTIFÍCIA UNIVERSIDADE CATÓLICA DE SÃO PAULO, BRASIL}

\begin{abstract}
RESUMO
Discute-se neste artigo, ao longo da apresentação de cinco experimentos, o caráter operante da resposta de observação, retomando-se a história deste conceito na literatura da análise experimental do comportamento. Reservase a expressão comportamento de observação para a relação entre uma resposta e suas conseqüências, e o termo resposta para as instâncias que produzem tal conseqüência. É destacada a necessidade de se levar em conta duas tríplices contingências no estudo do comportamento de observação e os cuidados que devem ser tomados ao chamarmos uma resposta como de observação. São discutidas as características que definem comportamento de observação e suas implicações no reforçamento condicionado e no controle de estímulos.

Palavras-chave: comportamento de observação, resposta de observação, reforçamento condicionado, controle de estímulos, dupla função de um estímulo
\end{abstract}

\begin{abstract}
This article examines the operant condition of observing behavior, briefly reviewing the history of this concept in the literature of experimental behavior analysis through the discussion of five experiments. The expression "observing behavior" is used to indicate the relation between a response and its consequences, and the term response for instances of the production of such consequences. The necessity of taking two three-term contingencies to study observing behavior and also the cautions that should be taken before calling a behavior an observing behavior are emphasized. The defining characteristics of an observing behavior and its implication in conditioned reinforcement and in stimulus control are discussed.

Key words: observing behavior; observing response; conditioned reinforcer; stimulus control; two functions stimulus
\end{abstract}

Wyckoff (1969)2 $)^{2}$ estudando aprendizagem de discriminação, define resposta de observação como "a resposta que resulta na exposição a um par de estímulos discriminativos" (p. 237). Como decorrência da afirmação de Wyckoff, a identificação de uma resposta como de observação depende da identificação da relação entre ela e as alteraçôes ambientais que ela produz; o que caracterizaria uma resposta como de observação seria a conseqüência que ela produz: "a exposição a um par de estímulos”. Para ressaltar isso, chamaremos essa relação entre a resposta e uma determinada conseqüência de comportamento de observação. Comportamento de observação, então, descreve a relação entre a emissão de uma resposta e a produção de conseqüências peculiares, a saber, conseqüências que têm função de estímulos discriminativos para outras respostas. Assim, quando estudamos comportamento de observação, estamos estudando uma relação na qual um mesmo estímulo participa de dois pro-

\footnotetext{
1 Artigo elaborado na vigência de Bolsa Produtividade CNPq para a segunda autora. Endereço para correspondência: Candido Pessôa, R. Monte Alegre, 1715, São Paulo, SP, CEP 05014-002.E-mail: candidopessoa@uol.com.br.

2 Este texto se refere a um experimento realizado em 1951, como parte dos requisitos para obtenção do grau de Doutor por Wyckoff. O ano de 1969 referese, por sua vez, à publicação do experimento em uma versão mais detalhada.
} 
cessos comportamentais: reforçamento condicionado e discriminação (Dinsmoor, Flint, Smith, \& Viemeister, 1969). O processo de reforçamento condicionado envolve o comportamento de observação e o processo de discriminação envolve o comportamento que será chamado aqui de comportamento de produção. Há, porém, um estímulo que ambos os comportamentos têm em comum. Este estímulo está entre duas respostas: uma resposta que o produz e que será chamada aqui de resposta de observação (RO) e uma resposta da qual ele é condição antecedente e que produz um reforçador específico e que será chamada aqui de resposta de produção (RP). A Figura 1 apresenta um esquema da relação entre estes dois comportamentos.

Ao se destacar a topografia da resposta de observação ao invés de sua função e, principalmente, ao não se considerar o papel das conseqüências produzidas pela resposta de observação - ou seja, da produção de estímulos discriminativos para a resposta de produção na geração e manutenção da resposta de observação, nós nos voltamos enganosamente para um "eu iniciador" como a origem da resposta de observação, afirmando-se, então,

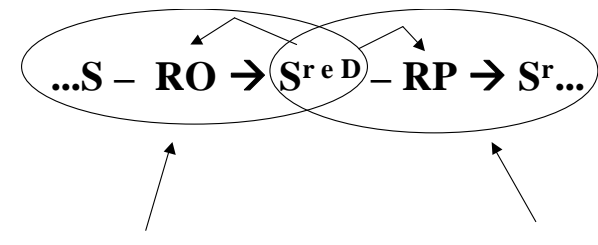

$$
\begin{aligned}
& \text { Contingência de } \\
& \text { observação } \\
& \text { (reforçamento } \\
& \text { condicionado) }
\end{aligned}
$$

$$
\begin{gathered}
\text { Contingência de } \\
\text { produção } \\
\text { (discriminação) }
\end{gathered}
$$

Figura 1. Diagrama das contingências envolvidas no estudo do comportamento de observação. RO é uma resposta de observação, RP é uma resposta de produção, $S^{\text {re D }}$ é um estímulo reforçador para a RO e discriminativo para a RP, o traço indica a relação entre a situação na qual a resposta é emitida e a emissão da resposta e a seta indica a relação entre a emissão da resposta e a produção do estímulo reforçador. uma capacidade do indivíduo de emitir respostas de observação à sua vontade.

Em alguns casos, mesmo quando as conseqüências produzidas pela resposta de observação são consideradas, passando-se a lidar com uma relação e não apenas com a topografia da resposta, os substantivos observação, atenção e percepção, assim como os verbos observar, atentar e perceber têm sido usados para definir, indicar ou referir-se a apenas uma das contingências descritas na Figura 1, ou seja, para dar ênfase a uma das funções do estímulo entre a RO e a RP. Dar ênfase a apenas uma das duas tríplices contingências descritas na Figura 1 também pode facilitar uma interpretação do comportamento que não seja totalmente desprendida de um "eu iniciador", pois, ao desconsiderar a contingência de produção, deixamos de indicar a origem do possível valor reforçador das conseqüências produzidas pela resposta de observação. E isto parece ser indispensável para uma descrição behaviorista radical do comportamento que procurará mostrar como o ambiente gera e mantém respostas de observação emitidas pelo indivíduo, destacando o caráter operante destas respostas, ao descrever eventos que são produzidos pela resposta de observação (Holland, 1958).

\section{A Resposta de Observação Como Uma Resposta Operante}

A demonstração do caráter operante de respostas de observação parece colocar exigências adicionais. A descrição dos eventos que são produzidos por tais respostas depende $\mathrm{da}$ identificação da própria resposta e, como destacam Dinsmoor (1983), Skinner (1953/ 1965) e Wyckoff (1952), essas respostas são, em muitos casos, encobertas ou de difícil 
mensuração, como por exemplo, a orientação dos órgãos sensoriais do indivíduo. Tais características da própria resposta de observação dificultam muito a tarefa do analista do comportamento em demonstrar o controle do ambiente sobre elas. Possivelmente, como forma de superar essa dificuldade, os pesquisadores que estudam o comportamento de observação incluem em seus experimentos respostas de observação arbitrárias que apresentam propriedades mais facilmente mensuráveis ou desenvolvem equipamentos especiais e sofisticados que tornam possível mensurar propriedades de respostas de difícil identificação.

Wyckoff (1969), estudando o processo de discriminação com pombos como sujeitos experimentais, delineou um experimento no qual a resposta de observação era pública e podia ser facilmente medida. Nesse delineamento, a resposta de observação (pressão em um pedal) transformava um esquema misto FI 30s EXT em um esquema múltiplo FI 30s EXT, isto é, com componentes iguais ao esquema anterior, porém, diferencialmente relacionados a estímulos do ambiente, ou seja, em um esquema no qual a resposta de observação produz estímulos discriminativos para a resposta de produção (bicar uma chave). Deve ser ressaltado que, neste caso, emitir a resposta de observação não altera o número de reforçadores que a resposta de bicar a chave poderia produzir, sendo possível ao sujeito experimental produzir todos os reforçadores emitindo somente a resposta de produção, independentemente da emissão de respostas de observação. Este procedimento ajudou a responder à questão de se apenas a produção de estímulos discriminativos para uma outra resposta (resposta de produção) teria função reforçadora em relação à $\mathrm{RO}$.
Em termos gerais, o experimento de Wyckoff (1969) consistiu de uma sessão preliminar de 45 minutos na qual pombos, privados de alimento, dentro de uma caixa de Skinner, foram expostos a uma chave iluminada com luz branca por 15 minutos e, nos 30 minutos seguintes, aleatoriamente iluminada com luz verde ou vermelha por períodos de 30 segundos. Durante essa sessão, bicadas na chave foram reforçadas com acesso a um comedouro em metade das vezes em que a chave estava iluminada com cada cor (branco, verde ou vermelho). Durante esta sessão foi registrado o tempo em que os pombos pressionaram um pedal localizado no chão da caixa, resposta para a qual, nesta condição, não havia nenhuma conseqüência programada. Após essa sessão preliminar, os pombos foram divididos em dois grupos. Para o grupo controle, nas cinco sessões seguintes, a produção de alimento por meio de bicadas na chave ocorria em um esquema misto FI 30s EXT, com componentes de 30 segundos de duração, que se alternavam aleatoriamente. Pressões no pedal localizado no chão da caixa iluminavam a chave com luz vermelha ou verde pelo tempo que durasse esta pressão, sem que estas cores estivessem relacionadas aos componentes em vigor. Para os pombos do grupo experimental, as pressões no pedal iluminavam a chave, consistentemente, com luz vermelha durante os componentes FI e com luz verde durante os componentes EXT; durante o tempo em que o pedal estava pressionado, o esquema em vigor mudava de misto para múltiplo.

Além dos pombos do grupo experimental apresentarem responder diferenciado em relação aos componentes em vigor, ao comparar o tempo de pressão no pedal do grupo experimental com o tempo de pressão no pedal 
do grupo controle, Wyckoff (1969) verificou que o pedal ficou um tempo maior da sessão pressionado no grupo experimental em relação ao grupo controle. Mesmo reconhecendo que, no grupo experimental, a resposta de pressão no pedal produzia duas conseqüências (luz vermelha, relacionada ao FI 30s e luz verde, relacionada à EXT) e mesmo sem ter analisado separadamente o tempo em que pressão no pedal produzia luz vermelha ou verde, o autor defende a interpretação de que a luz vermelha, quando sistematicamente relacionada ao componente FI, havia adquirido função de reforçador condicionado da resposta de observação.

Em 1958, Holland, investigando as variáveis que controlam a vigilância em humanos, delineou uma tarefa experimental na qual os participantes, militares colocados em uma sala escura, deveriam detectar movimentos de um ponteiro em um visor. Para a detecção ser possível, o participante deveria pressionar um botão, que iluminava o visor por 0,07 segundo (após este breve período, o botão precisava ser solto e novamente pressionado para o visor ser novamente iluminado por mais 0,07 segundo). Os movimentos dos ponteiros no visor foram programados para ocorrer de modo contingente a pressões neste mesmo botão sob diversos esquemas de reforçamento. Holland denominou a resposta de pressão neste botão de resposta de observação. $\mathrm{O}$ experimentador programou para essa resposta contingências em FI, FR, mult FR FI, DRL e VI com contenção limitada (limited hold) e os resultados deste experimento mostram que o esquema de reforçamento em que os sinais eram apresentados determinou a frequiência e distribuição das $\mathrm{RO}$ emitidas pelos participantes. Assim, pode-se dizer que o movimento dos ponteiros no visor controlou a taxa ou a probabilidade de emissão das respostas de observação e, além disso, que este controle é operante.

Schroeder e Holland (1968) avançaram no estudo da resposta de observação na análise do comportamento ao lidarem com os movimentos sacádicos dos olhos ${ }^{3}$ que, conforme afirmam os autores, é uma resposta de observação "natural". Os pesquisadores delinearam um experimento em que movimentos dos olhos de três estudantes universitários ficaram sob controle de esquemas de reforçamento em DRL 10s, FR 45, FI 120s e mult DRL FR FI. Para demonstrar o controle operante de movimentos sacádicos dos olhos, os autores montaram um painel quadrado de madeira com quatro visores, um próximo a cada vértice do quadrado. Cada visor continha um ponteiro que se mexia de acordo com contingências programadas pelos pesquisadores. Os três estudantes foram colocados, um por vez, durante várias sessões de aproximadamente 20 minutos de duração, diante do painel. Foi pedido a eles que relatassem, por meio de pressão em um botão, toda vez que o ponteiro de um dos visores se movesse. Utilizando um equipamento que registrava a posição dos olhos dos participantes durante as sessões, os pesquisadores mediram a freqüência acumulada dos movimentos sacádicos dos olhos dos participantes em direção aos visores, a duração das fixaçôes do olhar em cada visor e o tempo entre o movimento de um dos ponteiros e o relato deste movimento pelo participante. Os ponteiros foram programados para mexer de

\footnotetext{
3 Os movimentos sacádicos são um tipo dentre os vários movimentos que os olhos humanos, assim como os de várias outras espécies, podem realizar. Para se ter uma idéia de como são estes movimentos basta estender os braços para frente, em posição perpendicular ao corpo, com o dedo indicador de cada mão levantado, a uma distância de aproximadamente 40 centímetros entre eles. Fixe o olhar em apenas um dos dedos e depois apenas no outro. O movimento extremamente rápido que ocorre entre as duas fixações chama-se movimento sacádico dos olhos (Schwartz, 2004).
} 
maneira contingente ao movimento dos olhos dos participantes em direção aos mostradores. Assim, por exemplo, durante o FI 120s, o ponteiro de um dos mostradores, programado aleatoriamente entre os quatro, se mexia quando o participante olhasse pela primeira vez para qualquer um dos mostradores após 120 segundos da última vez que um ponteiro tivesse se movido ou, durante o FR 45, um ponteiro somente se mexia após o participante olhar um total de 45 vezes para os mostradores. As curvas de freqüência acumulada dos movimentos dos olhos obtidos por Schroeder e Holland (1968) são similares às curvas típicas de esquemas de DRL, FR, FI e mult DRL FR FI, evidenciando que os movimentos sacádicos dos olhos das pessoas podem ser controlados pelo esquema de reforçamento em vigor.

Pode-se dizer que os experimentos relatados até aqui (Wyckoff, 1969; Holland, 1958; Schroeder e Holland, 1968) demonstram, com respostas de diferentes topografias e com maior ou menor facilidade de identificação, como a resposta de observação é reforçada pela produção de estímulos discriminativos para a resposta de produção; são essas relações que constituem comportamentos geralmente nomeados como de atenção, de observação ou de "olhar para”. É interessante destacar que essa caracterização do reforçamento da resposta de observação está em acordo com a caracterização que Skinner (1957a/1999) fez ao discutir outro experimento da mesma série de experimentos de Holland (Holland, 1957): "Qualquer ato que leve um organismo a contatar um estímulo discriminativo, ou a clarificar ou intensificar seu efeito, é reforçado por este resultado e deve ser explicado em tais termos." (p. 157). Essa mesma posição é apresentada por Skinner em seu livro Verbal Behavior quando, ao analisar formas de construir novas respostas verbais, ele destaca a importância da função discriminativa do estímulo que é produzido pela resposta de observação: "Qualquer comportamento é reforçado se clarificar ou intensificar o efeito de um estímulo que satisfaz uma função discriminativa importante" (Skinner, 1957b/ 1992, p. 416). O termo “importante", na citação acima, pode revelar um aspecto fundamental para a análise que está sendo apresentada: talvez seja da importância da função discriminativa do estímulo que derive seu valor como reforçador condicionado.

Essas afirmações de Skinner e os experimentos relatados até este ponto destacam o caráter operante da resposta de observação, descrevendo como esta foi gerada e mantida pelas suas conseqüências. Mas fazem mais, caracterizam as conseqüências produzidas pela resposta de observação como tendo a função de estímulo discriminativo para outras respostas, o que, necessariamente, caracteriza a resposta de observação como aquela que é instalada e mantida por reforçadores condicionados. Entretanto, o próprio Skinner (1953/1965) parece reconhecer uma outra possibilidade para o entendimento da instalação e manutenção de tais respostas. Skinner (1953/1965), ao discutir reforçadores generalizados, afirma que:

Uma forma de comportamento precorrente pode preceder diferentes tipos de reforçamento em diferentes ocasiōes. A estimulação imediata de tal comportamento vai, então, se tornar um reforçador generalizado. Nós somos automaticamente reforçados, à parte de qualquer privação específica, quando controlamos o mundo físico com sucesso. [...] É possível, entretanto, que algo do efeito reforçador do 'retorno sensorial' (sensorial feedback) seja incondicionado. Um bebê 
parece ser reforçado por estimulação do ambiente que não foi seguida de reforçamento primário. [...] A capacidade de ser reforçado desta maneira poderia ter surgido durante o processo evolucionário, e pode ter paralelo no reforçamento que recebemos do simples "fazer o mundo se comportar”. (p. 77-78).

A partir desse trecho de Skinner (1953/ 1965), podemos levantar a possibilidade de que a resposta de observação seja gerada e mantida pela produção de reforçadores condicionados de um tipo especial, isto é, generalizados ou mesmo que seja reforçada por um reforçador incondicionado específico que poderíamos chamar de "fazer o mundo se comportar"

\section{A FUNÇÃO DA RESPOSTA DE OBSERVAÇÃO NO}

\section{CONTROLE DE ESTÍMULOS}

Até aqui, procurou-se mostrar que a resposta de observação é reforçada por suas conseqüências, isto é, é uma resposta operante. Tendo como base tal proposição, podemos estudar as relações entre a resposta de observação e o estabelecimento do controle de estímulos discriminativos para uma outra resposta. Para isso, serão apresentados dois experimentos: o primeiro, realizado por Madden e Perone (1999), e o segundo, por Dube e McIlvane (1999).

Madden e Perone (1999) realizaram um experimento sobre sensibilidade a esquemas de reforçamento em intervalo variável com componentes de diferentes valores em esquemas concorrentes, com três mulheres adultas, que envolveu cinco condições experimentais. $\mathrm{Na}$ primeira condiçãao, em cada sessão de 20 minutos, as participantes foram colocadas diante de um monitor de vídeo, um mouse, um botão "coletor de reforços" preto, e um botão vermelho, que deveria ficar pressionado durante todo o tempo da sessão, ou, caso contrário, a sessão era interrompida e só reiniciava quando esse botão era novamente pressionado. A tela do monitor era dividida ao meio e havia um pequeno quadrado branco em cada lado. Quando o cursor estava sobre um dos quadrados, um clique (RP), produzia pontos em esquema VI, posteriormente, trocados por dinheiro. No início de uma sessão, o cursor estava aleatoriamente colocado em um dos lados da tela e para mudança do cursor de um lado da tela para o outro era necessário clicar em outro quadrado, disposto na parte de cima da tela. Cliques neste quadrado superior mudavam o lado da tela em que estava o cursor após três segundos (atraso para mudança COD - de três segundos).

Os VI vigentes nos quadrados de produção de pontos eram sempre apresentados aos pares (nas sessões da Fase 1, VI 65s e VI 10s ou VI 65s e VI 410s; nas sessões da Fase 2, VI 65 s e VI 26s ou VI 65s e VI 164s) e, durante uma sessão, os dois VI mudavam de lado aleatoriamente. Assim, durante uma sessão, havia quatro possibilidades de disposição dos VI na tela (por exemplo, nas sessões da Fase 1, VI 65s à direita e VI 10s à esquerda, VI 10s à direita e VI 65s à esquerda, VI 65s à direita e VI 410s à esquerda e VI 410s à direita e VI 65s à esquerda). A disposição e o tempo de vigência de cada VI em cada posição foram alternados de forma que fosse difícil às participantes discriminarem, pelo tempo decorrido da sessão, qual VI estava em vigor de cada lado da tela do

4 Estudos na análise do comportamento têm destacado a relação entre resposta de observação e reforçamento condicionado. O estudo de um dos conceitos tem conduzido ao outro. Para uma revisão desta literatura veja Dinsmoor (1995), Fantino (1977) e Tomanari (2000). 
computador. Porém, as alternações eram balanceadas de forma que cada VI ficasse pelo mesmo tempo à direita e à esquerda. Não havia estímulo, além dos reforços, que indicasse qual VI estava em vigor em cada lado da tela.

Após a taxa de respostas de produção de pontos em cada um dos VI da Fase 1 estabilizar, as participantes foram expostas a sessões com os pares de VI da Fase 2 até que a taxa de respostas em cada VI desta fase também se estabilizasse. Os autores verificaram que a taxa de respostas não era distribuída de acordo com a proporção de reforços recebidos em cada VI, como seria previsto pela Lei da Igualação, (B1/ $\mathrm{B} 2)=\mathrm{k}(\mathrm{R} 1 / \mathrm{R} 2)^{\mathrm{a}}$, quando a constante "a" é igual a um ${ }^{5}$. Ao invés disso, as participantes emitiram aproximadamente o mesmo número de respostas em cada VI, independentemente de quantos reforços eram obtidos em cada VI ("undermatching"), indicando pouca sensibilidade ao esquema (ou seja, o parâmetro "a" da Lei da Igualação foi menor que um).

Uma segunda condição foi colocada às participantes, na qual uma resposta de observação, emitida em um outro quadrado na parte superior da tela, fazia com que, por 10 segundos, aparecesse uma cor, relacionada ao VI vigente, em cada quadrado de produção de pontos. Nesta condiçãa, duas participantes emitiram poucas respostas de observação, concentradas nas primeiras sessões e não alteraram o padrão de distribuição de respostas ("undermatching"). A terceira participante emitiu respostas de observação durante toda a fase e passou a clicar apenas sobre o quadrado que tinha maior taxa de reforçamento entre os dois VI disponíveis a cada momento ("overmatching").
Na terceira condição, o ponto só era ganho após a participante selecionar corretamente a cor relacionada ao VI do qual este ponto era proveniente, indicando uma entre três cores disponíveis num menu que aparecia na tela do computador. Nesta condição, as três participantes passaram a emitir respostas de observação e a ter a alocação de respostas em cada VI na proporção da taxa de reforçamento de cada VI ("matching”). A identificação correta das cores em relação aos esquemas aconteceu entre $96 \%$ e $99 \%$ das vezes.

Numa quarta condição, foi eliminada a exigência de indicação da cor relacionada ao VI do qual o ponto era proveniente para a liberação deste ponto, mas a $\mathrm{RO}$ continuava disponível (replicação da segunda condição). Nesta fase, as participantes continuaram a observar (uma participante em taxa menor que anteriormente) e mantiveram a sensibilidade próxima a um, ou seja, continuaram a igualar a proporção de respostas em cada VI à proporção da taxa de reforçamento.

Finalmente, em uma quinta condição que replicava a primeira condição, foi suspensa a possibilidade de emissão de $\mathrm{RO}$, e a taxa de respostas emitida em cada VI voltou a ser aproximadamente a mesma, independentemente das diferentes taxas de reforçamento em vigor a cada momento, ou seja, as participantes voltaram ao "undermatching". Ao discutirem os resultados obtidos em seu experimento, Madem e Perone (1999) destacam o aumento $\mathrm{da}$ sensibilidade às diferentes taxas de reforçamento em esquemas concorrentes quando a resposta de observação, que produz diferentes estímulos relacionados aos diferentes esquemas disponíveis, é possibilitada ou

5 Segundo Madden e Perone (1999), na fórmula acima, "B1" e "B2" são a quantidade de comportamento alocado durante cada VI, "R1" e "R2" são as taxas de reforçamento durante cada VI, "k" é o viés na tendência de se alocar respostas em um dos lados da tela e "a" é a sensibilidade à quantidade de reforços de cada VI. 
requerida. Os autores também destacam que o fato de apenas uma de três participantes emitir respostas de observação durante a segunda condição experimental pode ser indicativo de que humanos emitem poucas respostas de observação em situações em que tais respostas não são imprescindíveis à obtenção de reforçadores por outra resposta (de maneira análoga ao procedimento proposto por Wyckoff, 1969), o que pode trazer implicações para o responder em esquemas concorrentes. Madden e Perone (1999) finalizam seu relato sugerindo que a emissão de respostas de observação é um componente importante da sensibilidade humana a esquemas concorrentes de reforçamento.

Vale ainda notar que a segunda condição do experimento de Madden e Perone (1999) apresenta um aspecto interessante em relação à resposta de observação: o fato de duas participantes emitirem poucas respostas de observação quando estas respostas não eram imprescindíveis à obtenção das fichas pode indicar que não houve a construção de uma história de reforçamento diferencial em relação às diferentes cores, ou seja, as participantes recebiam pontos na presença ou ausência da cor relacionada ao determinado esquema. Esta ausência de história de reforçamento diferencial, possivelmente ligada à pequena "importância" da cor em relação ao recebimento das fichas, pode ter impedido que as diferentes cores se tornassem estímulos discriminativos para o responder diferencial das participantes. Já na terceira condição, as respostas de observação eram imprescindíveis, como no delineamento de Holland (1958), ou seja, a exigência de indicar a cor presente quando o ponto era produzido tornou obrigatória a produção da cor ou, em outras palavras, a participante era obrigada a emitir a resposta de observação. Assim, as participantes passaram a viver uma história de reforçamento diferencial em relação às diferentes cores. $\mathrm{Na}$ condição seguinte, quando esta história já estava construída, as participantes continuaram a emitir as respostas de observação, uma vez que as luzes já teriam adquirido função de estímulos reforçadores condicionados.

Um segundo experimento que faz uso do conceito de resposta de observação no estabelecimento do controle dos estímulos discriminativos foi realizado por Dube e McIlvane (1999). Os autores tratam da superseletividade de estímulos: um controle de estímulos atipicamente limitado com respeito à extensão, amplitude ou número de características do estímulo ou estímulos e que geralmente afeta o desempenho em tarefas de igualação ao modelo, em classes de educação especial (Dube et al., 2003). Dube e Mcllvane (1999) investigaram a possibilidade de que a superseletividade estivesse relacionada ao que os autores chamam de "não observação" de todas as características de um estímulo necessárias para a produção de reforçadores. Para isso, realizaram a pesquisa com três indivíduos com desenvolvimento atípico em tarefas de igualação com o modelo atrasado, realizadas em uma tela de computador sensível ao toque.

A pesquisa teve três fases. $\mathrm{Na}$ primeira fase, uma linha de base, um estímulo modelo, composto de duas figuras de formas arbitrárias, aparecia na tela do computador. Ao tocar o estímulo modelo, este desaparecia da tela e, simultaneamente, três estímulos de comparação simples surgiam (três figuras, sendo que apenas uma era igual a uma das duas que faziam parte do estímulo modelo). Se o participante tocasse, então, o estímulo de comparação igual a uma das figuras que compunham 
o estímulo modelo, recebia fichas, posteriormente trocadas por salgadinhos de sua preferência. Os participantes responderam no estímulo comparação correto, isto é, o que era uma das figuras do estímulo modelo, em aproximadamente dois terços das tentativas a que foram submetidos (supostamente, acertavam ao redor de $100 \%$ das vezes quando uma das figuras aparecia como comparação e aproximadamente 33\% quando a outra figura aparecia), o que, segundo os autores, indica que havia superseletividade.

A segunda fase envolveu sessões com dois tipos intercalados de tentativas num total de 42 tentativas. Um tipo de tentativa, realizada seis vezes por sessão, consistia numa igualação com o modelo por identidade sem atraso: havia a apresentação de um modelo composto de duas figuras; ao tocar o modelo, este permanecia na tela e três estímulos de comparação também compostos de duas figuras apareciam, sendo que, em um estímulo de comparação, as duas figuras que o compunham eram iguais às do modelo e, nos outros dois, apenas uma das figuras era igual a uma das figuras do modelo e a outra era diferente. Se o participante tocasse no estímulo comparação igual ao estímulo modelo, recebia fichas. O segundo tipo de tentativa, realizada 36 vezes por sessão, consistia da soma da tarefa anterior à tarefa da primeira fase. A tentativa começava com a apresentação de um modelo composto de duas figuras; ao tocar o modelo, este permanecia na tela e três estímulos compostos apareciam, sendo que apenas um era exatamente igual ao modelo. Ao tocar qualquer um dos três estímulos comparação, os três desapareciam da tela, permanecendo apenas o estímulo modelo e nenhuma conseqüência diferencial ocorria. $\mathrm{Na}$ mesma tentativa, ao tocar o modelo novamente, este desaparecia e apareciam três estímulos de comparação simples, sendo que só um dos três era igual a uma das duas figuras que compunham o estímulo modelo. Se o participante tocasse o estímulo comparação cuja figura era igual a uma das que compunham o modelo, ele era reforçado. Dube e McIlvane (1999) relatam que, com este procedimento, o efeito de superseletividade desapareceu, ou seja, os três participantes responderam no estímulo comparação considerado correto em mais de 90\% das tentativas nas sessões desta fase.

Na terceira e última fase do experimento, houve um retorno à condição de linha de base, ou seja, a tarefa a que os participantes foram submetidos foi igual à da primeira fase. $\mathrm{O}$ resultado desta terceira fase foi o reaparecimento da superseletividade, isto é, os participantes voltaram a responder no estímulo comparação simples considerado correto em aproximadamente dois terços das tentativas.

Os resultados de Dube e McIlvane (1999) mostram que a função de estímulo discriminativo das conseqüências produzidas pela resposta de observação é crucial para a definição de resposta de observação. É comum chamarmos de resposta de observação, em tarefas de igualação ao modelo, a resposta de tocar o estímulo modelo quando esta produz os estímulos comparação. Porém, "produção", no caso deste experimento, pode não se referir à inclusão dos estímulos no ambiente do participante, ou seja, os estímulos que apareceram na tela do computador podem ter sido incluídos no ambiente dos pesquisadores, porém, não no ambiente dos participantes. É assim que pode ser interpretado o fato dos participantes não reagirem diferencialmente a uma das figuras do estímulo composto, quando expostos à situação na qual, após a apresentação 
do modelo composto, deviam "escolher", entre três estímulos simples, aquele que era uma das figuras que compunham o estímulo modelo. Os participantes só reagiam diferencialmente diante de uma das figuras componentes, a outra não controlava o responder dos participantes. Apenas depois do reforçamento diferencial que teve como critério a emissão de respostas na presença das duas figuras que compunham o estímulo modelo (as seis tentativas de igualação ao modelo por identidade sem atraso que aconteciam como parte das tentativas que constituíram a segunda condição experimental), os experimentadores conseguiram que uma resposta que antes não era obrigatória (olhar para as duas figuras do estímulo modelo) fosse emitida, eliminando, assim, o efeito que chamaram de superseletividade.

Com isso pode-se voltar à definição de resposta de observação apresentada no início deste texto. Tem-se, aí, talvez, um dos melhores exemplos de como respostas não são adequadamente definidas por meio de suas características topográficas. A mesma forma de resposta - tocar o estímulo modelo composto - só poderia ser chamada de resposta de observação quando produzia estímulos que tinham função discriminativa para respostas de escolha (que são, neste caso, as respostas de produção); isto parece evidenciar que as conseqüências produzidas pela resposta são aspectos definidores de respostas de observação. Para ressaltar este fato, talvez seja melhor falar em comportamento de observação no lugar de resposta de observação, uma vez que o termo comportamento já inclui, pelo menos, a relação resposta-conseqüência.

A série de estudos apresentada procurou ilustrar o caráter operante da resposta de observação, independentemente do tipo de reforço que possa estar controlando esta resposta: incondicionado, condicionado ou condicionado generalizado. $\mathrm{O}$ fato da resposta de observação poder ser prescindível, como no caso dos experimentos de Wyckoff (1969) e de uma das fases do experimento de Madden e Perone (1999), talvez seja um dos fatores que facilitam interpretações de que as emissões deste tipo de resposta não dependem do ambiente. Levar em conta a dupla função do estímulo produzido pela resposta de observação pode contribuir para uma compreensão mais clara das condiçôes que controlam o comportamento humano e, também, para um planejamento mais adequado de ambientes tanto experimentais como aplicados, desafios constantes dos analistas do comportamento.

\section{REFERÊNCIAS}

Dinsmoor, J. A., Flint, G. A., Smith, R. F., \& Viemeister, N. F. (1969). Differential reinforcing effects of stimuli associated with the presence or absence of a schedule of punishment. In D. P. Hendry (Ed.). Conditioned Reinforcement, (pp. 357-384). Homewood, IL: The Dorsey Press.

Dinsmoor, J. A. (1983). Observing and conditioned reinforcement. The Behavioral and Brain Sciences, 6, 693-728.

Dinsmoor, J. A. (1995). Stimulus control: Part I. The Behavior Analyst, 18, 51-68.

Dube, W. V., Lombard, K. M., Farren, K. M., Flusser, D. S., Balsamo, L. M., Fowler, T. R., \& Tomanari, G. Y. (2003). Stimulus oversectivity and observing behavior in individuals with mental retardation. In S. Soraci Jr., \& K. Murata-Soraci (Eds.). Visual information processing, (pp.109-124). Westport, MA: Praeger Publishers.

Dube, W. V., \& McIlvane, W. J. (1999). Reduction of stimulus overselectivity with nonverbal differential 
observing responses. Journal of Applied Behavior Analysis, 32, 25-33.

Holland, J. G. (1957). Technique for behavioral analysis of human observing. Science, 125, 348-350.

Holland, J. G. (1958). Human vigilance. Science, 128, 61-63.

Madden, G. J., \& Perone, M. (1999). Human sensitivity to concurrent schedules of reinforcement: Effects of observing schedule-correlated stimuli. Journal of the Experimental Analysis of Behavior, 71, 303-318.

Schroeder, S. R., \& Holland, J. G. (1968). Operant control of eye movements. Journal of Applied Behavior Analysis, 1, 161-166.

Schwartz, S. H. (2004). Visual perception - A clinical orientation. New York: McGraw-Hill.

Skinner, B. F. (1953/1965). Science and human behavior. New York: The Free Press.

Skinner, B. F. (1957/1992). Verbal Behavior. Acton:
Copley Puplishing Group.

Skinner, B. F. (1957/1999). The experimental analysis of behavior. In B. F. Skinner. Cumulative record definitive edition, (pp.132-164). Acton, MA: Copley Puplishing Group.

Tomanari, G. Y. (2000). Reforçamento condicionado. Revista Brasileira de Terapia Comportamental e Cognitiva, 2, 61-77.

Wyckoff, L. B., Jr. (1952). The role of observing responses in discrimination learning. Psychological Review, 59, 431-442.

Wyckoff, L. B., Jr. (1969). The role of observing responses in discrimination learning. In D. P. Hendry (Ed.). Conditioned reinforcement, (pp. 237260). Homewood: The Dorsey Press.

Submetido em 12 de fevereiro de 2006 Aceito em 4 de dezembro de 2006 


\section{ACTA COMPORTAMENTALIA}

É uma publicação semestral dedicada à difusão das contribuições científicas da análise do comportamento no âmbito das línguas de origem latina. Publica trabalhos originais em espanhol, italiano, francês e português.

ACTA COMPORTAMENTALIA inclui os seguintes tipos de artigos:

a) propostas teóricas

b) análises conceituais e históricas

c) estudos experimentais sobre o comportamento animal e humano

d) investigaçōes avaliativa e tecnológica sobre comportamento animal e humano

e) relatos técnicos

f) comunicaçōes científicas

g) revisões bibliográficas em profundidade

Os números 1 e 2, com número especial de aniversário, do volume 15 da ACTA COMPORTAMENTALIA serão lançados, respectivamente, em Junho e Dezembro de 2007.

Informaçōes:

Luz Adelina Félix R.

ACTA COMPORTAMENTALIA

Apdo. Postal No. 5-374

Col. Chapalita

45030 Guadalajara,Jal.

México

E-mail:Ifelix@cencar.udg.mx 